\title{
Evaluation of the WHO Clinical Case Definition of AIDS in the Indian Context: Suggesting a Prediction Rule
}

\author{
P. Kaushik ${ }^{a}$ R.M. Pandey ${ }^{\mathrm{b}}$ J.N. Pande ${ }^{\mathrm{a}}$ \\ Departments of a Medicine and ${ }^{b}$ Biostatistics, All India Institute of Medical Sciences, \\ New Delhi, India
}

\section{Key Words}

Clinical case definition . Human

immunodeficiency virus infection . Acquired immunodeficiency syndrome $\cdot$ India

\begin{abstract}
Objective: To test the statistical significance of the existing World Health Organization (WHO) clinical case definition for the diagnosis of acquired immunodeficiency syndrome (AIDS) in areas where diagnostic resources are limited in India, and frame a prediction rule that is sensitive and specific for the clinical diagnosis of AIDS. Methodology and Results: In a case-load study, we evaluated the WHO clinical case definition of AIDS in 162 patients, the training set (58 seropositives) at the All India Institute of Medical Sciences, New Delhi. This definition had a sensitivity of about $29 \%$, specificity of about $29 \%$, positive
\end{abstract}

predictive value of about $19 \%$ and negative predictive value of about $42 \%$. We analyzed the clinical data of these 162 patients using stepwise logistic regression analysis. Generalized lymphadenopathy, oropharyngeal candidiasis, presumptive esophageal candidiasis, herpes zoster in the last 5 years and a total lymphocyte count of less than 1,000 $\times$ $10 \% / \mathrm{lemerged}$ as important predictors. A prediction rule for the diagnosis of AIDS in the Indian context was then developed empirically using the coefficients of these predictors. We further tested the rule prospectively in 50 patients, the test set (11 seropositives) and found it to have a sensitivity of $81.8 \%$, specificity of $97.4 \%$, positive predictive value of $90 \%$, and a negative predictive value of 95\%. Conclusion: The WHO definition was not significant statistically for clinical use in India. The new prediction rule may help in AIDS surveillance in India.

Copyright $@ 2000$ S. Karger AG, Basel

Dr. Prashant Kaushik, MD, Clinical-Fellow in Rheumatology and

Immunology, University of Texas Southwestern Medical Center Dallas, TX 75235-8884 (USA)

Tel. +1 972756 9348, Fax +1 $2146483011 / 2146487995$

E-Mail kaushikprashant@hotmail.com 


\section{Introduction}

Studies of the natural history of human immunodeficiency virus (HIV) infection have documented a wide spectrum of disease manifestations, ranging from asymptomatic infection to life-threatening conditions characterized by severe immunodeficiency, serious opportunistic infections and malignancies. The strong, and at times, nearly exclusive association of some of these conditions (individually or together) with a diagnosis of acquired immunodeficiency syndrome (AIDS) has frequently helped clinicians to identify previously unsuspected HIV infection.

In April 1986, the first evidence of HIV infection in commercial sex-workers was reported from Madras in India [1]. Subsequent countrywide surveillance has shown its widespread prevalence throughout the country [24]. Although HIV-1 is the predominant infecting agent there is evidence that HIV-2 may also be more prevalent than hitherto appreciated [5]. Various surveys in India have shown that the important population groups at risk for HIV infection are female commercial sex-workers, promiscuous heterosexuals, professional (paid) blood donors and the intravenous drug users (IVDU) in the three North Eastern states of Manipur, Mizoram and Nagaland [6-8].

The need of a clinical case definition (CCD) for AIDS was felt by public health agencies to monitor diseases resulting from HIV infection. An ideal CCD should be sensitive, specific, simple, inexpensive, consistent over time to project future trends and consistent between nations to allow international comparisons. The World Health Organization (WHO) CCD [9], though simple and inexpensive, has not been evaluated in India. The 1993 revised Centers for Disease Control CCD [10] is impractical in India because the sophisticated investigations required for the confirmatory diagnosis of many opportunistic conditions are not freely available. Also, it has been suggested that the clinical spectrum of AIDS in tropical countries may be different from that in the developed nations [11].

The present study was undertaken with the objectives of evaluating the sensitivity, specificity and predictive values of the WHO CCD in patients suspected of AIDS and for developing a prediction rule based on simple criteria for the diagnosis of AIDS in subjects of unknown HIV status.

\section{Method}

Till the end of 1996, 3,080 cases of AIDS had been reported in the country [12]. The study was conducted from February 1993 to February 1997 at the hospital of the All India Institute of Medical Sciences (AIIMS), which is a tertiary care center catering to a large number of patients for complicated diagnoses and management from all parts of India. The patient population comprised of all the patients referred to the medical outpatient department of AIIMS over this period. The sample for the study was a total of 329 new patients seen on fixed days by the principal investigator (P.K.) in the medical outpatient department during this 4year period. A written informed consent for history, examination and investigations was obtained from 162 patients, who were enrolled in the study. Patients who were known to be immunocompromised due to any malignancy, diabetes mellitus, steroids, immunosuppressant drugs etc. were not considered for the study. All the enrolled patients were evaluated according to a standard questionnaire to gather information about demography (age and sex), sexual history (whether homosexual, heterosexual or bisexual, if there was a history of contact with commercial sex-workers, promiscuity and the use of barrier contraceptives during these contacts), a history of blood transfusion (if so, whether from paid or voluntary donors) or of being an IVDU, the three major and the five minor criteria of the WHO CCD and additional criteria, namely presumptive esophageal candidiasis (PEC), modified Folstein's Mini Mental State examination score (MMS), tuberculosis (TB), presumptive Pneumocystis carinii pneumonia (PPCP), tuberculin test status and total lymphocyte count (TLyC, see 'Appendix' for the definitions of the various parameters studied). 
The investigator was not aware of the HIV serological status of the patients at the time of enrollment. Like most other validation studies we studied the individuals at a single point in time. Due to limited diagnostic facilities and technical constraints, necropsies were not done.

The serum of these patients was subjected to enzyme-linked immunosorbent assay (ELISA) using Genelavia mixt test kit (Sanofi Diagnostics Pasteur, France). It was tested in triplicate supplemented by the Western blot IgG assay (Diagnostic Biotechnology Pte Ltd., Singapore). The CD4+ and CD8+ lymphocytes were enumerated by Coulter Immunoprep Epics leukocyte preparation system (Coulter Corp., Hialeah, Fla., USA). HIV seropositivity (3 times positive ELISA with a positive Western blot) was used as the reference standard.

The WHO CCD was evaluated on these 162 patients (training set). Univariate analysis was then performed on the various clinical and laboratory parameters. The parameters found to be statistically significant $(\mathrm{p}<0.05)$ were subjected to logistic regression using a stepwise approach. Using the coefficients of those parameters that ultimately remained in the mod$\mathrm{el}$, a prediction rule was framed. This is somewhat similar to a previous study [13]. This prediction rule was tested over 50 patients (test set) selected from the same source, i.e. the medical outpatient department of the AIIMS, in the same way, i.e. all the 50 consecutive patients seen by the principal investigator (P.K.) after the initial 162 patients of the 'training set', to yield its sensitivity, specificity and predictive value.

\section{Results}

All the 212 patients (162 training set and 50 test set) who enrolled in the study underwent complete evaluation. A total of 69 patients (58 for training set and 11 for test set) were seropositive for HIV infection and clinically symptomatic. Forty-seven were males while 22 were females. Among the seropositive patients $78 \%$ were either commercial sexworkers or had experienced sex with commercial sex-workers (all had multiple partners and unprotected intercourse). Of these 1 patient was bisexual. No patient was purely homosexual. Fifteen percent of the seropositive pa-
Table 1. Evaluation of the WHO CCD in 162 patients (the training set, 58 seropositives)

\begin{tabular}{cccc}
\hline \multicolumn{4}{c}{ HIV serology } \\
\cline { 2 - 4 } & positive & negative & total \\
\hline WHO CCD satisfied & & \\
Yes & 17 & 74 & 91 \\
No & 41 & 30 & 71 \\
\cline { 2 - 4 } & 58 & 104 & 162 \\
\hline
\end{tabular}

Sensitivity $29.3 \%$, specificity $28.8 \%$, positive predictive value $18.7 \%$, negative predictive value $42.3 \%$.

tients had received blood transfusions from professional donors, $3 \%$ were IVDU while 3\% denied any clue to the source of HIV infection.

Twenty-six percent of the AIDS patients died during hospital stay. No malignancies (including invasive cervical cancer, Kaposi's sarcoma, lymphoma) could be diagnosed in any patient with AIDS.

The results obtained by applying the WHO CCD to the 162 patients (the training set) are shown in table 1 . The statistical evaluation of the various parameters is shown in tables 2 and 3.

The model formed after logistic regression analysis and its statistical evaluation is shown in table 4. PEC, generalized lymphadenopathy (GL), herpes zoster (HZ), oropharyngeal candidiasis (OC) and a TLyC of less than 1,000 $\times$ $10^{9} / 1$ were the five parameters that remained in the model. Using their coefficients, a prediction rule was developed, which is shown in table 5. A cutoff score of 17 was selected based on the results obtained after applying the rule on the 162 patients of the 'training set'. On evaluating this rule prospectively over further 50 patients (test set), 11 of whom were seropositive for HIV, it had a high sensitivity, specificity and predictive value (table 6). 
Table 2. Univariate analysis of the parameters $(n=162$, the training set)

\begin{tabular}{|c|c|c|c|c|}
\hline \multirow{2}{*}{ Parameter } & \multicolumn{2}{|c|}{ HIV serology } & \multirow[t]{2}{*}{$\mathrm{p}$ value } & \multirow{2}{*}{$\begin{array}{l}\text { Odds ratio } \\
(95 \% \mathrm{CI})\end{array}$} \\
\hline & positive & negative & & \\
\hline \multicolumn{3}{|l|}{ Chronic fever } & 0.62 & $0.84(0.43-1.66)$ \\
\hline Present & 38 & 72 & & \\
\hline Absent & 20 & 32 & & \\
\hline \multicolumn{3}{|l|}{ Weight loss } & 0.26 & $1.48(0.75-2.93)$ \\
\hline Present & 41 & 65 & & \\
\hline Absent & 17 & 39 & & \\
\hline \multicolumn{3}{|l|}{ Diarrhea } & 0.02 & $2.2 \quad(1.15-4.22)$ \\
\hline Present & 33 & 39 & & \\
\hline Absent & 25 & 65 & & \\
\hline \multicolumn{3}{|l|}{$P E C$} & 0.001 & $3.42(1.73-6.76)$ \\
\hline Present & 41 & 43 & & \\
\hline Absent & 17 & 61 & & \\
\hline \multicolumn{3}{|l|}{$H Z$} & 0.001 & $5.14(2.52-10.47)$ \\
\hline Present & 31 & 19 & & \\
\hline Absent & 27 & 85 & & \\
\hline \multicolumn{3}{|l|}{$O C$} & 0.001 & $5.88(2.86-12.09)$ \\
\hline Present & 32 & 18 & & \\
\hline Absent & 26 & 86 & & \\
\hline \multicolumn{3}{|l|}{$G L$} & 0.001 & $3.19(1.64-6.20)$ \\
\hline Present & 34 & 32 & & \\
\hline Absent & 24 & 72 & & \\
\hline \multicolumn{3}{|l|}{$G U$} & 0.01 & $2.66(1.32-5.35)$ \\
\hline Present & 26 & 22 & & \\
\hline Absent & 32 & 72 & & \\
\hline \multicolumn{3}{|l|}{$P P C P$} & 0.001 & $4.87(2.28-10.40)$ \\
\hline Present & 25 & 14 & & \\
\hline Absent & 33 & 90 & & \\
\hline \multicolumn{3}{|l|}{$M M S<25 / 30$} & 0.001 & $4.86(2.41-9.80)$ \\
\hline Present & 32 & 21 & & \\
\hline Absent & 26 & 83 & & \\
\hline \multicolumn{3}{|l|}{$T B$} & 0.01 & $0.34(0.17-0.66)$ \\
\hline Present & 27 & 75 & & \\
\hline Absent & 31 & 29 & & \\
\hline \multicolumn{3}{|c|}{ Tuberculin test negativity } & 0.001 & $11.07(5.21-23.51)$ \\
\hline Present & 44 & 23 & & \\
\hline Absent & 14 & 81 & & \\
\hline \multicolumn{3}{|l|}{$T L y C$} & 0.001 & $9.24(4.44-19.26)$ \\
\hline$<1,000 \times 10^{9} / 1$ & 42 & 23 & & \\
\hline$>1,000 \times 10^{9} / 1$ & 16 & 81 & & \\
\hline \multicolumn{3}{|c|}{$G D$} & 0.001 & $0.15(0.08-0.31)$ \\
\hline Present & 16 & 74 & & \\
\hline Absent & 42 & 30 & & \\
\hline \multicolumn{3}{|c|}{ Chronic cough (without TB) } & 0.01 & $0.41(0.20-0.78)$ \\
\hline Present & 18 & 55 & & \\
\hline Absent & 40 & 49 & & \\
\hline
\end{tabular}

The significant odds ratios are in italics; for the definitions of parameters refer to the Appendix. 
Table 3. Evaluation of the parameters for the training set $(\mathrm{n}=162)$

\begin{tabular}{llll}
\hline Parameter & Sensitivity, $\%$ & Specificity, $\%$ & PPV, $\%$ \\
\hline Chronic fever & 65.5 & 30.8 & 34.5 \\
Weight loss & 70.7 & 37.5 & 38.7 \\
Chronic diarrhea & 56.9 & 63.5 & 45.8 \\
PEC & 70.7 & 58.7 & 48.8 \\
HZ & 53.4 & 81.7 & 62.0 \\
OC & 55.2 & 82.7 & 64.0 \\
GL & 58.6 & 69.2 & 51.5 \\
GU & 44.8 & 69.2 & 54.2 \\
PPCP & 43.1 & 86.5 & 64.1 \\
MMS $<25 / 30$ & 55.2 & 79.8 & 60.4 \\
TB & 46.6 & 27.9 & 26.5 \\
Tuberculin anergy & 75.9 & 77.9 & 65.7 \\
TLyC <1,000 $\times 10^{9} / 1$ & 72.4 & 77.9 & 64.6 \\
GD & 27.6 & 28.8 & 17.8 \\
Chronic cough & 31.0 & 47.1 & 24.7 \\
\hline
\end{tabular}

$\mathrm{PPV}=$ Positive predictive value
Table 4. Logistic regression analysis $\left(n=162\right.$, pseudo $R^{2}=0.88$, training set)

\begin{tabular}{lrlrl}
\hline Parameter & OR & Coefficient & \multicolumn{1}{l}{$95 \% \mathrm{CI}$} & $\mathrm{p}$ value \\
\hline PEC & 137 & 4.92 & $2.21-7.64$ & 0.000 \\
GL & 35 & 3.57 & $0.79-6.34$ & 0.012 \\
HZ & 33 & 3.51 & $0.58-6.44$ & 0.019 \\
TLyC & 13 & 2.53 & $-0.01-5.08$ & 0.051 \\
OC & 11 & 2.40 & $-0.18-4.97$ & 0.698 \\
\hline
\end{tabular}

Sensitivity $96.3 \%$, area under curve 0.9822 , specificity $97.2 \%$. $\mathrm{CI}=$ Confidence interval; $\mathrm{OR}=$ odds ratio.

\section{Discussion}

The study gives an idea regarding the descriptive epidemiology of AIDS in India. The sensitivity, specificity and predictive value of the WHO CCD were lower as compared to the results in the developing countries of Africa [14-16]. There was no significant age difference between the patients with AIDS and without AIDS. The predominant modes of infection were heterosexual promiscuous unprotected contacts, blood transfusion from paid donors and IVDU, which is in conformity with previous studies [6-8].

It also shows that a wide spectrum of disease in association with underlying HIV immunosuppression exists in the country. Of all the WHO CCD's criteria, fever and weight 
Table 5. Scoring system

\begin{tabular}{lc}
\hline Parameter & Score \\
\hline OC & 5 \\
TLyC $<1,000 \times 10^{9} / 1$ & 5 \\
HZ in last 5 years & 7 \\
GL & 7 \\
PEC & 10 \\
\cline { 2 - 2 } Total & 34
\end{tabular}

Prediction rule: Cutoff score more than or equal to 17.

Sensitivity $91.2 \%$, specificity $95.1 \%$, positive predictive value $87.3 \%$, negative predictive value $96.7 \%$.

Table 6. Evaluation of the prediction rule on test set $(\mathrm{n}=50)$

\begin{tabular}{llc}
\hline Observed & \multicolumn{2}{l}{ Classified (HIV serology) } \\
\cline { 2 - 3 } & positive & negative \\
\hline Positive & 9 & 1 \\
Negative & 2 & 38 \\
\hline
\end{tabular}

Sensitivity $81.8 \%$, specificity $97.4 \%$, positive predictive value $90 \%$, negative predictive value $95 \%$.

loss were the two most prevalent major criteria. This is similar to the presentation shown in a previous study in India [17]. However chronic fever, diarrhea and weight loss were poor markers for AIDS by stepwise logistic regression analysis. This is because even in seronegative patients, the various tropical causes of these symptoms were widely prevalent. GL and generalized dermatitis (GD) were also not impressive parameters. $\mathrm{HZ}$ and OC both had a high specificity for diagnosing AIDS, although their positive predictive values were moderate. The combination of OC with dysphagia or retrosternal pain thought to be a good predictor of esophageal involvement [18] (PEC) was a fairly sensitive criterion. Genital ulceration (GU) suggestive of herpes simplex infection had a tendency for chronicity (average duration of 2 months) and recurrence (in 16 out of the 26 AIDS patients) as described previously [19]. Chronic cough was a poor marker for AIDS, as shown in Africa also [20]. Cryptococcal meningitis per se was not indicative of AIDS. This has been shown in a previous Indian study too [17]. This diagnosis was made in 7 patients, 4 of whom were seronegative. Although mentioned that most patients with AIDS and pulmonary cryptococcosis have disseminated disease unlike the situation in non-AIDS patients in whom Cryptococcus tends to localize to the lungs [21], our single patient of disseminated cryptococcosis (lungs, liver, pericardium, meninges) was repeatedly negative on testing for HIV. Impaired concentration, memory and other cognitive functions as symptoms of AIDS dementia complex (which was a clinical diagnosis based on the MMS score) were seen in a significant number of patients of AIDS. This parameter had a fair positive predictive value with a good specificity. One AIDS patient had a spontaneous pneumothorax but neither Pneumocystis carinii was isolated, nor aerosolized pentamidine was given to this patient. $P$. carinii has been detected in AIDS patients in India [22]. The insidious onset of $P$. carinii pneumonia allows significant pulmonary parenchymal destruction leading to the development of cysts, cavities, bullae and pneumothorax. The poor sensitivity, specificity and positive predictive value of TB denotes that it cannot be considered as an AIDS-defining disease in India [cf. Centers for Disease Control revised definition 1993, ref. 10]. Anergy to tuberculin, which was seen in a majority of the patients of AIDS, has been reported earlier also [23, 24]. A large proportion of the Indian population is tuber- 
culin-positive by adolescence. Hence anergy to this antigen in the absence of any obvious cause of immunosuppression should alert the physician towards an underlying HIV infection. Oral hairy leukoplakia was not seen in any patient of AIDS. This is probably because the Epstein-Barr virus infections are not so frequent in India. Similarly Kaposi's sarcoma was also not present in any patient. This malignancy is extremely rare in our country.

Among the AIDS patients the mean CD4+ lymphocyte count was $86 \times 10^{9} / 1$. Ninety percent of the patients had a count less than 200 $\times 10^{9} / 1$. All the patients had a CD4/CD8 ratio of less than 0.5 . There was a strong positive correlation (Pearson correlation coefficient $>0.4$ ) between CD4+ lymphocyte count and TLyC. TLyC is a simple test and had a good sensitivity, sepcificity and positive predictive value. It has even been considered a surrogate marker for CD4+ lymphocyte counts earlier [25].

No patient who was seronegative in the first test was found to be positive in further tests. However literature reports that up to $19 \%$ of the HIV-1 ELISA-negative patients can be positive for HIV either by Western blot, HIV antigen or viral culture [26].

Data from this study also show that sexually transmitted diseases may be a major public health problem in our population. Hence it is an indication for assigning a high priority to sexually transmitted disease interventions in ongoing AIDS control efforts.

Using logistic regression analysis (table 4), OC, TLyC, HZ, GL, and PEC were found to be statistically significant parameters. The coefficients of these parameters were used to assign scores to each one of them. This algorithm had fair sensitivity, specificity and predictive values if a 'cuttoff' score of 'more than or equal to 17' was used to identify the AIDS cases on the 'training set' (table 5). The same algorithm (prediction rule) when evaluated on the 'test set' of 50 patients was found to have fairly good statistical significance (table 6).

\section{Limitations of the Study}

Firstly, some criteria of the WHO CCD were modified for the purpose of this study. For example, chronic disseminated herpes simplex genitalis was diagnosed on the basis of superficial mucosal ulcerative lesions of the genitalia (painful, nonhealing, recurrent or spreading). This was done because the definition itself does not specify how these criteria should be applied. Secondly, five out of the nine criteria of the WHO CCD incorporate specific duration or frequencies which are difficult to elicit accurately from many patients. Thirdly, in order to reduce the semantic discrepancies between Western medical terms and their nearest vernacular equivalents, translation and lack of familiarity among respondents with some concepts could have caused some misclassification or bias. Fourthly, it was also difficult and unreliable to elicit a history of homosexuality due to associated social stigma in India. Fifthly, we have evaluated the CCD using HIV serological status as the reference standard. This underestimates the sensitivity of the CCD because a CCD will only identify individuals with clinical symptoms and/or signs while serological testing detects asymptomatic cases as well. Sixthly, the generalization of the prediction rule developed on hospital patients may not extend to the general population. Lastly a larger data set may be required for the validation of this rule.

\section{Conclusion and Recommendations}

The overall performance of the WHO CCD was not satisfactory. A prediction rule for evaluating the probability of AIDS in a patient suspected of this disease on the basis of simple clinical and laboratory features has 
been developed. This rule may be of use to help select the patients requiring tests for HIV infection and opportunistic conditions.

We therefore recommend that larger studies be conducted in other parts of the country to provide more information regarding the clinical presentation of AIDS in these areas and the efficacy of the prediction rule. Priorities must be set in the use of available resources. Discussion should focus on how reliable HIV testing can be made available on a larger scale, how supplemental or confirmatory testing can be undertaken in a valid and cost-effective manner and how ethical standards can be guaranteed in all work entailing HIV testing. Health care workers must be trained in using this prediction rule to diagnose AIDS. Lastly AIDS case reporting should be improved in India.

\section{Appendix}

1 Bisexual: A person who had experienced sexual contact in any form with people of the same and opposite sex.

2 Chronic cough (without tuberculosis): Dry or productive cough lasting for $>1$ month.

3 Chronic diarrhea: At least two stools per day, of unusually loose consistency for at least 30 days over preceding 2 months or less.

4 Chronic fever: Fever of any grade; intermittent or constant; of duration more than 1 month.

5 Commercial sex-workers: Those individuals who received money for their sexual contacts in any form.

6 Cryptococcal meningitis: Clinical features of meningitis with a positive India-ink preparation in cerebrospinal fluid.

7 Disseminated tuberculosis: Histopathological evidence of caseating granulomas on a specimen from two noncontiguous sites with or without positive staining for acid-fast bacilli.

8 Generalized lymphadenopathy: On examination, lymph nodes palpable at two or more noncontiguous, extrainguinal locations $>1 \mathrm{~cm}$ in size. The groups examined were occipital, preauricular, postauricular, cervical, supraclavicular, axillary and epitrochlear.
9 Generalized dermatitis: Itchy, ill-defined papular eruptions, diffusely distributed.

10 Genital ulceration: Superficial mucosal ulcerative lesions (anal or genital); painful, nonhealing, recurrent or spreading.

11 Herpes zoster: A history of painful or dysaesthetic prodrome followed by cutaneous vesicular eruptions, unilateral or bilateral, recurrent or encrusted, in the last 5 years or on examination scarred marks suggestive of the same.

12 Heterosexual: A person who had experienced sexual contact in any form with another person of opposite sex.

13 Homosexual: A person who had experienced sexual contact in any form with another person of the same sex.

14 Intravenous drug user: A person who gave a history of intravenous injections permitting exposure to HIV-infected blood.

15 Kaposi's sarcoma: A characteristic gross appearance of suspicious erythematous or violaceous plaque-like lesion on skin or mucous membranes. As per Centers for Disease Control guidelines, since it is infrequent in India to see a case of Kaposi's sarcoma, its presumptive diganosis was not made.

16 Modified Folstein's Mini Mental State Examination:

a Orientation in time

5

(day, date, month, year, time)

b Orientation in place

(hospital, storey, city, state, country)

c Repeat three words (pen, table, lamp) 3

d Backward counting of five numbers 5

e Recall the three words in (c) 3

f Recognize and name 'pen' 2

g Three-stage verbal command to follow: 3

Take the paper in right hand

Fold it in half

Put it on the floor

h Read a written command and follow

i Copy the design

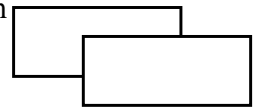

If all sides and angles are preserved

17 Oropharyngeal candidiasis: White curd-like membrane or patches on the palate, tongue, buccal mucosa or pharyngeal wall which could be scraped off with wooden spatula to reveal a punctate erythematous mucosal surface. 
18 Pneumocystis carinii pneumonia (presumptive): A history of dyspnea on exertion, chest pain or nonproductive cough of recent onset (within the past 3 months) and diffuse bilateral interstitial infiltrates on chest X-ray with no evidence of a bacterial pneumonia (presence of cyanosis, tachypnea or tachycardia strengthened the diagnosis).

19 Presumptive esophageal candidiasis: Recent onset of retrosternal pain on swallowing along with oral candidiasis.

20 Promiscuous: A person who had sexual contact in any form with more than one partner.

21 Protected/unprotected sexual contact: This was decided by the use of a barrier contraceptive by either of the partners.

22 Pulmonary tuberculosis: Chest-radiological evidence of apical infiltrates, miliary mottling or cavity with or without positive sputum staining for acid-fast bacilli.
23 Total lymphocyte count: Percentage of lymphocytes (on differential leukocyte count) $\times$ total leukocyte count.

24 Tuberculin test: $0.1 \mathrm{ml}$ of purified protein derivative was injected intradermally on volar aspect of forearm and the size of induration read after $72 \mathrm{~h}$ as $\leq 10 \mathrm{~mm}$ : negative response; $\geq 10 \mathrm{~mm}$ : positive response.

25 Weight loss: Involuntary loss of more than $10 \%$ of the baseline body weight over preceding 6 months or less. As accurate measurements were usually not available, this was often based on history and qualitative observations rather than by direct weighing.

\section{References}

1 John TJ, George Babu PG, Jayakumari $\mathrm{H}$, Simoes EA: Prevalence of HIV infection in risk groups In Tamil Nadu, India (letter). Lancet 1987;i:160-161.

2 Malaviya AN, Singh RR, Khare SD, et al: AIDS screening in North India: Clinical spectrum of HIV infection. J Assoc Physicians India 1987; 35:405-410.

3 ICMR Bull: Turning point in HIV/ AIDS research: Vision of hope. Dec 1996, No 12.

4 Kamat HA, Banker DD: Human immunodeficiency virus-1 infection among patients with sexually transmitted diseases in Bombay. Natl Med J India 1993;6:11-13.

5 Rubsamen-Waigmann H, Briesen H, Maniar JK, Rao PK, Scholz C, Pfutzner A: Spread of HIV-2 in India (correspondence). Lancet 1991; 337:550-551.

6 Lal S: Current status of AIDS and $\mathrm{HIV}$ in India. J Indian Med Assoc 1992:12-13.

7 Singh YN, Chandra M, Nanu A, Bhasin R, Kapoor S, Malaviya AN: HIV infection in the blood donors of Delhi, India: 1 1/2 years' experience. J Acquire Immune Defic Syndr 1991;4:1008-1009.
8 Sarkar S: Rapid spread of HIV among injecting drug users in North Eastern states of India. Bull Narc 1993;45:91-105.

9 World Health Organization: Acquired immunodeficiency syndrome (AIDS), WHO/CDC case definition for AIDS. Wkly Epidemiol Rec 1986;61:69-72.

10 Centers for Disease Control: 1993 revised classification system for HIV infection and expanded surveillance case definition for AIDS among adolescents and adults. MMWR Morb Mortal Wkly Rep 1992;41:1-19.

11 Quinn TC, Mann JM, Curran JW, Piot P: AIDS in Africa: An epidemiologic paradigm. Science 1986;234: 955-963.

12 National AIDS Control Organization: Monthly Update on HIV Infection in India. Ministry of Health and Family Welfare Government of India, Nov 1996.

13 Weniger BG, Quinhoes EP, Sereno $\mathrm{AB}$, et al: A simplified surveillance case definition of AIDS derived from empirical clinical data. J Acquir Immune Defic Syndr 1992;5: 1212-1223.
14 Widy-Wirski R, Berkley S, Downing R, Okware S, Recine U, Mugerwa R, Lwegaba A, Sempala S: Evaluation of the WHO clinical case definition for AIDS in uganda. JAMA 1988; 260:3286-3289.

15 Colebunders R, Francis H, Izaley L, et al: Evaluation of a clinical casedefinition of acquired immunodeficiency syndrome in Africa. Lancet 1987;i:492-494.

16 Colebunders RL, Braun MM, Nzila N, Dikilu K, Muepu K, Ryder R: Evaluation of the WHO clinical case definition of AIDS among tuberculosis patients in Kinshasa, Zaire (letter). J Infect Dis 1989;160:902903.

17 Kaur A, Babu PG, Jacob M, Narasimhan C, Ganesh A, Saraswathi NK, Mathai D, Pulimood BM, John TJ: Clinical and laboratory profile of AIDS in India. $\mathbf{J}$ Acquir Immune Defic Syndr 1992;5:883-889.

18 Bonacini M, Young T, Laine L: The causes of esophageal symptoms in human immunodeficiency virus infection: A prospective study of 110 patients. Arch Intern Med 1991; 151:1567-1572. 
19 Bagdades EK, Pillay D, Square SB, O’Neill CO, Johnson MA, Griffiths PD: Relationship between herpes simplex virus ulceration and CD4+ cell counts in patients with HIV infection. AIDS 1992;6:1317-1320.

20 Gilks CF, Otieno LS, Brindle RJ, et al: The presentation and outcome of HIV-related disease in Nairobi. QJM 1992;82:25-32.

21 Clark RA, Greer DL, Valainis GT, Hyslop NE: Cryptococcus neoformans pulmonary infection in HIV-1 infected patients. J Acquir Immune Defic Syndr 1990;3:480-484.
22 Singh YN, Singh S, Rattan A, et al: Pneumocystis carinii infection in patients of AIDS in India. J Assoc Phys India 1993;41:41-42.

23 Pape JW, Liautaud B, Thomas F, Mathurin JR, St Amand MM, Boncy M, Pean V, Pamphila M, Laroche AC, Johnson WD Jr: Characteristics of the acquired immunodeficiency syndrome (AIDS) in Haiti. N Engl J Med 1983;309:945-950.

24 Van De Perre P, Rouvroy D, Lepage P, Bogaerts J, Kestelyn P, Kayihigi J, Hekkes AC, Butzler JP, Clameck $\mathrm{M}$ : Acquired immunodeficiency syndrome in Rwanda. Lancet 1984; ii:62-65.
25 Martin DJ, Sim JGM, Sole J, et al: CD4+ lymphocyte count in African patients coinfected with HIV and tuberculosis. J Acquir Immune Defic Syndr 1995;8:386-391.

26 Colebunders R, Francis H, Duma MM, Van der Groen G, Lebughe I, Kapita B, Quinn TC, Heyward WL, Piot P: HIV-1 infection in HIV-1 enzyme-linked immunoassay seronegative patients in Kinshasa, Zaire. Int J STD AIDS 1990;1:330-334. 